\title{
Cyclic interconnection for formation control of 1-D vehicle strings
}

\author{
Andrés A. Peters ${ }^{\mathrm{a}, *}$, Richard H. Middleton ${ }^{\mathrm{b}}$, Oliver Mason ${ }^{\mathrm{c}}$ \\ a Advanced Center for Electrical and Electronic Engineering, Universidad Técnica Federico Santa María, Valparaíso, Chile \\ ${ }^{\mathrm{b}}$ Centre for Complex Dynamic Systems and Control, The University of Newcastle, Callaghan NSW 2308, Australia \\ ${ }^{\mathrm{c}}$ Hamilton Institute, National University of Ireland Maynooth, Maynooth, Co. Kildare, Ireland
}

\section{A R T I C L E I N F O}

\section{Article history:}

Received 18 November 2014

Received in revised form

30 October 2015

Accepted 2 December 2015

Recommended by A. Astolfi

Available online 12 December 2015

Keywords:

Cyclic formation control

Interconnected systems

Linear Systems

Stability

String stability

\begin{abstract}
A B S T R A C T
In this paper, we study a formation control scheme for a 1-D string of vehicles. Each member tracks the movement of its immediate predecessor but also the first vehicle tracks the position of the last member of the string. We discuss conditions for the stability of the full interconnected system and show that if a constant spacing policy is used, the stability of the system is lost after the string size exceeds a certain number depending on the model parameters (vehicles and controllers). Additionally, we study the use of a constant time headway spacing policy. If the associated time headway parameter is greater than a critical value, the interconnected system is stable and string stable for any string size. Finally, we show that if an independent leader vehicle is added to the formation and every follower has access to this leader position, the cyclic formation with a constant spacing policy can be made stable and string stable by appropriately selecting a design parameter.
\end{abstract}

(c) 2015 European Control Association. Published by Elsevier Ltd. All rights reserved.

\section{Introduction}

Formation control of autonomous vehicles has received great attention in recent decades $[11,3,2,21,15]$. Due to its importance in applications to automated highway systems, the simple case of a 1-D platoon of linear vehicles has been studied extensively and researchers have considered diverse alternatives to achieve coordinated movement of the string (see $[19,12,5]$ and the references therein).

A simple control strategy is to equip every member of the formation with a compensator that stabilizes its position in closed loop, using as a reference the position of its predecessor on the string and a desired inter-vehicle spacing. This approach achieves a tight formation in steady state for a constant speed of the leader vehicle. More elaborate approaches consider also using the states of the lead vehicle and/or the states of other members of the formation (see for example $[8,6,12]$ and the references therein).

The first element of interest when studying such architectures is the stability of the full interconnected system. The authors of Fax and Murray [4] discuss the effect of the information flow in vehicle formations and how the eigenvalues of the graph Laplacian matrix play an important role when determining stability. Other aspects

\footnotetext{
* Corresponding author. Tel.: +3531 7086100; fax: +35317086269.

E-mail addresses: andres.peters@usm.cl (A.A. Peters),

Richard.Middleton@newcastle.edu.au (R.H. Middleton),

oliver.mason@nuim.ie (O. Mason).
}

of importance in the architecture are those of performance and safety. Works such as Seiler et al. [19], Middleton and Braslavsky [15], Barooah and Hespanha [1] describe how in interconnections that are stable, disturbances may be amplified along the formation resulting in poor performance or even collisions (string instability).

The present work studies a particular interconnection where the first vehicle in the formation tracks the position of the last member, while the rest of the members track the position of their immediate predecessors. This interconnection is cyclic and allows the members of the formation to compensate disturbances at any location of the string of vehicles. This is in contrast to unidirectional architectures where the leader moves independently; in such scenarios a follower does not detect or manoeuvre in response to disturbances that affect members behind it. There are a few interesting applications for such models. In particular, this can correspond to an idealized ring road in large cities (where the physical curvature of the road can be considered negligible for the effects of the vehicle's trajectories). This interconnection can also be a model for subway or railway/tram lines with a circular layout. Moreover, in some cities, bus routes of public transport are normally composed of bus lanes, where particular vehicles are not allowed to circulate. The buses travel from point A to B but they normally return from $B$ to A, essentially becoming a cyclic flow of vehicles. Although these trajectories are performed in a 2-D space, overtaking in such scenario is not a common practice and turns in road junctions could be considered as disturbances. In such a 
setting, every vehicle travels almost exclusively in a straight line, similar to travelling on tracks. Nevertheless, the vehicles need not be necessarily arranged in a 2-D ring, they can be moving along a straight road and at the same time have a cyclic interconnection.

Recent works have investigated a similar setting. In Marshall et al. [13] the authors study formations of vehicles in cyclic pursuit. In Lafferriere et al. [10] several formation control architectures using a graph theoretic approach are studied, focusing on stability. The authors of Roberson and Stilwell [17] discuss the use of a circulant communication network to simplify the estimation and design of a state feedback to control a vehicle platoon. In Park and Ahn [16] the authors discuss stabilization of directed cycle formations of agents moving in the plane.

The results reported in Rogge and Aeyels [18] motivate much of our work. We consider the same basic interconnection structure as this earlier paper. In particular, the authors considered a specific and simple model for every vehicle. Moreover, they selected constant controller gains and obtained conditions on the parameters in order to achieve stability and string stability of the interconnection. We consider extensions to this results, allowing more general vehicle models and controllers by using a frequency domain approach. We will show that if the spacing policy is constant, the interconnection is unstable after the formation size exceeds a certain critical number. Additionally, we show that if a time headway spacing policy is used, stability for any size of the formation and string stability can be achieved by choosing an appropriate value for the time headway parameter.

This paper also provides results for the addition of an independent leader vehicle which sends its location to every follower. In this case, the stability and string stability of the interconnection is related to the selection of the weight parameter that every follower uses to track its predecessor and the leader simultaneously. Similar results for non-cyclic cases are reported in Seiler et al. [19] and Shaw and Hedrick [20]. One simple practical example of the use of a leader corresponds to subway lines. The trains could receive the position of a fictitious leader in addition to using the position of its predecessor to control their trajectories. In other circumstances, the cyclic interconnection might be able to alleviate the relaying of the leader state to every member. In this case the largest distance to relay the state to every member would be half the distance of a unidirectional scheme (the centremost member would be the farthest in a communication sense from a leader that stays in front of the platoon).

The remainder of this paper is organized as follows. Section 2 gives some preliminary assumptions on the problem to be studied. Section 3 presents the cyclic control strategy and the associated dynamics. The main contributions of the paper are contained in Sections 4 and 5. Section 4 shows stability results for the two inter-vehicle spacing policies and Section 5 contains analogous results for the use of an independent leader vehicle. Some numerical examples and comments on the main results are given in Section 6. Section 7 contains some final remarks and possible lines of future work.

\section{Framework and problem formulation}

\subsection{Notation}

The notation used in this paper follows much of the standard systems and control literature. Lowercase is used for real scalar signals, $x: \mathbb{R} \rightarrow \mathbb{R}$ with specific values of the signal denoted by $x(t)$. Uppercase is used for scalar complex-valued Laplace transforms of signals and transfer functions, $X: \mathbb{C} \rightarrow \mathbb{C}$ with specific values denoted by $X(s)$. For the sake of brevity in the notation, where there is no confusion, the argument (s) will be omitted. Vectors

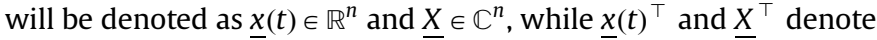
their transposes. Moreover, the all zeros and all ones vector will be denoted as 0 and 1 respectively. The imaginary unit is denoted by $j$, with $j^{2}=-1$. Boldface will be used for matrices $\boldsymbol{G} \in \mathbb{C}^{n \times m}$ and the $(i, k)$ th entry of $\boldsymbol{G}$ is denoted by $G_{i, k}$. The magnitude of $X$ when $s=j \omega, \omega \in \mathbb{R}$, is denoted by $|X|$ and its magnitude peak over all possible values of $\omega$ is denoted as ||$X||_{\infty}:=\sup _{\omega}|X(j \omega)|$. The derivative of $X(s)$ with respect to $s$ will be denoted as $X^{\prime}(s)$. For $z \in \mathbb{C}, \mathfrak{R}(z)$ and $\mathfrak{I}(z)$ denote the real and imaginary parts of $z$ respectively.

\subsection{Vehicle model, control strategy and initial conditions}

We consider a platoon of $N \in \mathbb{N}$ identical vehicles, with positions $z_{i}(0 t)$, initial positions $z_{i}(0)$ and initial velocities $\dot{z}_{i}(0)$ for $1 \leq i \leq N$, modeled by linear time invariant systems. In the frequency domain, the models of each member of the platoon are given by

$Z_{i}=P\left(U_{i}+D_{i}\right)+\frac{z_{i}(0)}{s}+\frac{\dot{z}_{i}(0)}{s^{2}}$ for $1 \leq i \leq N$,

where $Z_{i}$ denotes the Laplace transform of $z_{i}(t), U_{i}$ is the control action and $D_{i}$ is an input disturbance, both acting on the $i$ th member. The transfer function for the vehicle $P$ has a single pole at the origin and is strictly proper (this is a more general vehicle description that the one used in Rogge and Aeyels [18] and a commonly used assumption Jovanovic and Bamieh [8]).

Now, we define the separation errors as

$e_{i}(t)=z_{i-1}(t)-z_{i}(t)-\left(\varepsilon_{i}+h \dot{z}_{i}(t)\right), \quad$ for $i=2, \ldots, N$,

$e_{1}(t)=z_{N}(t)-z_{1}(t)-\left(\varepsilon_{1}+h \dot{z}_{1}(t)\right)$,

where $\varepsilon_{i} \in \mathbb{R}$, and $h \geq 0$ is the time headway parameter.

The control objective is to maintain the errors $e_{i}(t)$ defined in (2) equal to zero whenever possible, which would imply that the vehicles retain a desired formation. We will study the control strategy given by

$U_{i}=K E_{i}, \quad$ for $i=1, \ldots, N$,

where $K$ is a strictly proper controller assumed to have a single pole at the origin and $E_{i}$ are the Laplace transforms of the signals $e_{i}(t)$.

In steady state, that is for $t \rightarrow \infty$, we aim to have $e_{i}(t)=0$ and also $\dot{z}_{i}(t)=v_{p} \in \mathbb{R}$ for all $i=1, \ldots, N$. Here we follow a similar approach to the one used in Rogge and Aeyels [18] and we add all the right hand sides of (2) and substituting yields

$v_{p}=-\frac{1}{h N} \sum_{i=1}^{N} \varepsilon_{i}$.

For simplicity, we will set $\varepsilon_{i}=\varepsilon \in \mathbb{R}$ for $i=2, \ldots, N$ and therefore $\varepsilon_{1}=-h N v_{p}-(N-1) \varepsilon$. With this, we take initial conditions such that the formation is initially in steady state, that is $z_{i-1}(0)-z_{i}(0)$ $=\varepsilon+h \dot{z}_{i}(0)$ for $i=2, \ldots, N, z_{N}(0)-z_{1}(0)=\varepsilon_{1}+h \dot{z}_{1}(0)$ and $\dot{z}_{i}(0)=v_{p}$ for $i=1, \ldots, N$. Finally, we define the change of coordinates

$x_{i}(t)=z_{i}(t)-z_{i}(0)-v_{p} t, \quad$ for $i=1, \ldots, N$,

and substituting in (2) yields

$e_{i}(t)=x_{i-1}(t)-x_{i}(t)-h \dot{x}_{i}(t), \quad$ for $i=2, \ldots, N$,

$e_{1}(t)=x_{N}(t)-x_{1}(t)-h \dot{x}_{1}(t)$

With this, we will focus on the effect of the disturbances $D_{i}$ on the separation errors $E_{i}$ when the initial conditions and $\varepsilon_{i}$ are set to zero. In the following sections we will have

$E_{i}=X_{i-1}-X_{i}-\operatorname{sh} X_{i}, \quad$ for $i=2, \ldots, N$,

$E_{1}=X_{N}-X_{1}-\operatorname{sh} X_{1}$. 
The main difference with the basic approach Seiler et al. [19], where the leader moves independently, is that the first vehicle now tries to maintain a predefined spacing with respect to the last member of the platoon. Applications of such a configuration may include subway systems that run on circuits and ring roads around major cities. Nevertheless, the vehicles need not be necessarily arranged in a 2D ring, they can be moving along a straight road and at the same time have a cyclic interconnection.

One potential advantage of such a strategy is the fact that a disturbance at any vehicle will be detected and compensated by every member of the formation. This could provide an extra level of safety when compared to unidirectional strategies where a disturbance at any vehicle is not detected by its predecessors (which could possibly lead to collisions).

\section{Dynamics of the interconnected system}

With the control strategy defined in the previous section, the vehicle dynamics are given by

$\underline{X}=(\boldsymbol{I}-P K \boldsymbol{G})^{-1} P \underline{D}$,

where $\underline{X}=\left[\begin{array}{lll}X_{1} & \cdots & X_{N}\end{array}\right]^{\top}, \quad \boldsymbol{I}$ is the $N \times N$ identity matrix, $\underline{D}=$ $\left[\begin{array}{lll}D_{1} & \cdots & D_{N}\end{array}\right]^{\top}$ and $\boldsymbol{G} \in \mathbb{C}^{N \times N}$ is the interconnection matrix:

$\boldsymbol{G}=\left[\begin{array}{cccc}-Q & & & 1 \\ 1 & \ddots & & \\ & \ddots & \ddots & \\ & & 1 & -Q\end{array}\right]$

with $Q=1+h s$.

We aim to obtain explicit formulae for the vehicle positions. To do so, we must invert the matrix $\mathbf{I}-P K \boldsymbol{G}$ in (8). In unidirectional control strategies with a free leader Seiler et al. [19], Klinge and Middleton [9] the matrix to invert is lower triangular and normally has a straightforward inverse, yielding dynamics for the vehicle positions that are easy to study. In the present case $\boldsymbol{I}-P K \boldsymbol{G}$ is circulant and the resulting dynamics will not be straightforward to analyze as will be seen below.

In the following, to simplify the presentation, we will consider strictly proper controllers $K$ that satisfy $K=\tilde{K} / Q$, with $\tilde{K}$ having no zero at $s=-1 / h$. Let $T=P K Q /(1+P K Q)=P \tilde{K} /(1+P \tilde{K})=1-S$, and also $\Gamma=T / Q$. Then, we have

$\boldsymbol{I}-P K \boldsymbol{G}=K P\left[\begin{array}{cccc}\Gamma^{-1} & & & -1 \\ -1 & \ddots & & \\ & \ddots & \ddots & \\ & & -1 & \Gamma^{-1}\end{array}\right]$,

and the corresponding inverse (which can be checked by multiplying) is given by

$(\boldsymbol{I}-P K \boldsymbol{G})^{-1}=\frac{S}{1-\Gamma^{N}}\left[\begin{array}{ccccc}1 & \Gamma^{N-1} & \cdots & \Gamma^{2} & \Gamma \\ \Gamma & 1 & \ddots & & \Gamma^{2} \\ \vdots & \ddots & \ddots & \ddots & \vdots \\ \Gamma^{N-2} & & \ddots & 1 & \Gamma^{N-1} \\ \Gamma^{N-1} & \Gamma^{N-2} & \cdots & \Gamma & 1\end{array}\right]$

By the structure of the interconnection, this matrix is also circulant and therefore the response of the $n$th vehicle to a single disturbance on the $k$ th vehicle is the same as the response of the $n+l$ th vehicle to a single disturbance on the $k+l$ th vehicle (there is no leader). Moreover, as a consequence of the chosen interconnection, a single disturbance affects every vehicle in the string.

In particular we have that the inter-vehicle spacings when $D_{1}$ $\neq 0$ and $D_{i}=0$ for $i=2, \ldots, N$ are given by $E_{i}=X_{i-1}-Q X_{i}=F_{i}^{(N)} D_{1}$, with

$F_{i}^{(N)}=\frac{S P(1-T) \Gamma^{i-2}}{1-\Gamma^{N}}, \quad$ for $i=2, \ldots, N$

$E_{1}=X_{N}-Q X_{1}=\frac{S P\left(\Gamma^{N-1}-Q\right)}{1-\Gamma^{N}} D_{1}=F_{1}^{(N)} D_{1}$

It is important to note that the dynamics of the inter-vehicle spacings have a factor $\left(1-\Gamma^{N}\right)^{-1}$. The poles of this transfer function increase in number and change in location with an increase of the string size $N$. This is in great contrast with other unidirectional architectures where the pole locations are unaffected by $N$. In those cases the dynamics are usually powers of the complementary sensitivity function $T$ [19].

\section{Properties of the interconnected system}

In this section, we state the main results of the paper. In particular we analyze the roots of the equation $1-\Gamma^{N}=0$ and its role in the stability of the interconnection. The following result $[14,19]$ plays an important role in the study of several formation control strategies and it also does in the present case.

Lemma 1. Let $T$ be a real rational scalar function of the complex variable s. Suppose that $T(0)=1$ and also that $T$ is stable (analytic in the closed right half complex plane) and strictly proper. Then

$\int_{0}^{\infty} \ln |T(j \omega)| \frac{d \omega}{\omega^{2}} \geq \frac{\pi}{2} T^{\prime}(0)$.

In this particular setting, since $P$ and $K$ both possess a single pole at the origin, the transfer function $T$ satisfies $T(0)=1$ and $T^{\prime}(0)=0$. Lemma 1 then implies that $|T(j \omega)|>1$ for some $\omega>0$.

We will consider two of the most common spacing policies and will obtain stability conditions of the interconnection for both. The following result, taken from Klinge and Middleton [9], shows how the use of a time headway policy can impact the frequency response of the interconnection.

Proposition 1. Let $T=P \tilde{K} /(1+P \tilde{K})$, with $P$ being strictly proper and having a pole at the origin and $\tilde{K}$ is a proper controller with a single pole at the origin and no zero at $s=-1 / h$. Then, $T$ is a stable and strictly proper transfer function such that $T(0)=1, T^{\prime}(0)=0$ and ||$T||_{\infty}>1$. Moreover, there exists $h_{0}>0$ such that ||$T /\left.(1+h s)\right|_{\infty}$ $>1$ for $0 \leq h<h_{0}$ and $|T(j \omega) /(1+j h \omega)|<1$ for $\omega>0$, whenever $h>h_{0}$. A formula for $h_{0}$ is given by

$h_{0}=\sqrt{\sup _{\omega}\left(\frac{\left|\frac{P \tilde{K}}{1+P \tilde{K}}\right|^{2}-1}{\omega^{2}}\right)}$

We also have the following result for $h_{0}$ to be used in.

Lemma 2. The value $h_{0}$ defined in (15) satisfies

$2 h_{0}^{2} \geq\left.\frac{d^{2}}{d \omega^{2}}\left(\left|\frac{P \tilde{K}}{1+P \tilde{K}}\right|^{2}\right)\right|_{\omega=0}$.

Proof. This follows directly by using L'Hôpital's rule to compute the limit of the right hand side of (15) when $\omega \rightarrow 0$.

The final preliminary result needed for the derivation of the main results is given in the following lemma.

Lemma 3. Let $\Gamma$ be a stable and strictly proper transfer function such that ||$\Gamma||_{\infty}>1$. Then, there exists an interval $\left[\theta_{1}, \theta_{2}\right] \subset[0,2 \pi]$ with $\theta_{1} \leq \theta_{2}$ such that $1-e^{j \theta} \Gamma=0$ has solutions in the open right half 
plane when $\theta \in\left(\theta_{1}, \theta_{2}\right)$. Moreover, if there exists $\omega_{c}$ such that $\mid \Gamma(j$ $\left.\omega_{c}\right) \mid=1$ and $\left|\Gamma\left(j \omega_{c}\right)\right|^{\prime} \neq 0$, we have $\theta_{1}<\theta_{2}$.

Proof. See Appendix A.

Remark 1. In the following we will consider $\Gamma$ that satisfies: if there exists $\omega_{c}>0$ such that $\left|\Gamma\left(j \omega_{c}\right)\right|=1$, then $\left|\Gamma\left(j \omega_{c}\right)\right|^{\prime} \neq 0$.

\subsection{Stability analysis}

To check the stability of the transfer functions $F_{i}$ in (12) we note that $S P$ and $\Gamma=T / Q$ are stable provided that the controller $K$ is properly designed. We will now focus on the behavior of $(1-T) /($ $1-\Gamma^{N}$ ) which appears in $F_{i}$ for $i=2, \ldots, N$. The results are analogous for $F_{1}$ and we omit the details for the sake of simplicity in the exposition. We have that

$\Gamma^{N}-1=\prod_{k=0}^{N-1}\left(\Gamma-e^{j 2 k \pi / N}\right)$.

Note that this echoes the results derived in Fax and Murray [4], where the term $e^{j 2 k \pi / N}$ is directly connected to the eigenvalues of the graph Laplacian matrix for this interconnection. We have the following stability result.

Theorem 1. Let $T$ and $Q=1+$ hs be defined as in Proposition 1 , and let $\Gamma=T / Q$. Then, there exists $h_{0}>0$ such that the following holds:

1. if $h>h_{0}$, then $(1-T) /\left(1-\Gamma^{N}\right)$ has all of its poles in the open left half plane;

2. if $h<h_{0}$ then there exists $N_{c} \in \mathbb{N}$ such that for all $N>N_{c},(1-T) /$ $\left(1-\Gamma^{N}\right)$ has poles in the right half plane.

Proof. (1) If $h>h_{0}$, Proposition 1 states that $|\Gamma|<1$ for all $\omega>0$. We will show that $(1-T) /(1-\Gamma)$ is stable. First we study the possible poles at the origin. If we recall that $T(0)=1$ and $T^{\prime}(0)=0$ we have that $1-T$ has two zeros at $s=0$. Since $\Gamma$ is stable, $\Gamma(0)=1$ and $|\Gamma|<1$ for all $\omega>0$, we have that for all $c \in(0,1),|c \Gamma|<1$ for all $\omega \geq 0$. This in turn implies that $|1-(1-c \Gamma)|<1$ for $\omega \in \mathbb{R}$. Rouche's Theorem ensures that $1-c \Gamma$ and 1 have the same number of zeros in the closed right half plane. By continuity of the roots, $1-\Gamma$ only has unstable roots at the stability boundary and given that $|\Gamma|<1$ for all $\omega>0$ this root can only be located at $s=0$. Computing $\Gamma^{\prime}=T^{\prime} / Q-T Q^{\prime} / Q^{2}$ implies that $\Gamma^{\prime}(0)=-h$ and therefore $1-\Gamma$ has only one root at $s=0$ if $h>0$. Consequently $(1-T) /(1-\Gamma)$ is stable. It remains to show that $1 / \prod_{k=1}^{N-1}$ $\left(\Gamma-e^{j 2 k \pi / N}\right)$ has poles only in the left half plane. For this, we note that the Nyquist plot of $-e^{j 2 k \pi / N} \Gamma$ does not encircle the point $s=$ -1 for any value of $2 k \pi / N$ with $k=1, \ldots, N-1$. Given that $\Gamma$ is open loop stable $\left(1-e^{j 2 k \pi / N} \Gamma\right)^{-1}$ is also stable for $k=1, \ldots, N-1$.

2. If $h<h_{0}$, Proposition 1 states that ||$\Gamma \|_{\infty}>1$. We write

$\frac{1-T}{1-\Gamma^{N}}=\frac{1-T}{1-\Gamma} \prod_{k=1}^{N-1}\left(1-e^{j 2 k \pi / N} \Gamma\right)^{-1}$

where $\frac{2 k \pi}{N} \in(0,2 \pi)$ when $k=1, \ldots, N-1$. According to Lemma 3 , given that in this case $\left.|| \Gamma\right|_{\infty}>1$, there exists an interval $\left(\theta_{1}, \theta_{2}\right)$ such that $1-e^{j \theta} \Gamma$ has zeros in the right half plane when $\theta \in\left(\theta_{1}, \theta_{2}\right)$. Now, if $\frac{2 \pi}{N}<\frac{\theta_{2}-\theta_{1}}{2}$ there is at least one point of the sequence $\left\{\frac{2 k \pi}{N}\right\}$ for $k=1, \ldots, N-1$ that belongs to $\left(\theta_{1}, \theta_{2}\right)$. Therefore, for all $N>N_{c}=\left|\frac{4 \pi}{\theta_{2}-\theta_{1}}\right|+1$ the transfer function $(1-T) /(1-$ $\Gamma^{N}$ ) has poles in the right half plane. $\square$

Part (1) of the last theorem implies that the condition $|\Gamma|<1$ for all $\omega>0$ is sufficient for stability of the transfer functions $F_{i}$ in (12). Part (2) states that if the time headway parameter satisfies $0 \leq h<h_{0}$, there exists a critical number $N_{c}$ for which any interconnection with a string size greater than $N_{c}$ will be unstable.
Remark 2. If either $P$ or $K$ have a pole at $s=p$ with $\Re(p) \geq 0$ we have $T(p)=1$ and $S(p)=1-T(p)=0$ (since $T$ is stable). Now, for all $h$ we have $1-\Gamma(p)^{N}=1-(T(p) /(1+h p))^{N}=1-1 /(1+h p)^{N}$. This expression only vanishes for $h p=0$ (and this case is already covered in the proof). Therefore, there are no cancellations of the zero at $s=p$ of $S=1-T$ with possible right half plane zeros of $\left(1-\Gamma^{N}\right)$.

Remark 3. If $h=h_{0}$ it is possible to have $\Gamma\left(j \omega_{c}\right)=e^{j \theta}$ for some $\omega_{c}>0$. If this is the case, there could be a value of $k$ and $n$ such that $\Gamma\left(j \omega_{c}\right)-e^{j 2 k \pi / n}=0$, or equivalently $\theta=2 k \pi / N$ implying that there is a pair of pure imaginary complex poles at $\pm j \omega_{c}$.

\subsection{String stability analysis}

Now we show that the interconnection can also be made string stable. We understand string stability as having certain sequences of transfer functions from disturbances to errors with a uniform bound on their magnitude peak; this bound is also independent of the string length (see for example [15]). We have the following result.

Theorem 2. Let $\Gamma=T / Q$ with $T$ defined as in Lemma 1 with $T^{\prime}(0)$ $=0$ and $Q=1+h s$, with $h>h_{0}$ (with $h_{0}$ defined in Proposition 1). Consider $F_{i}^{(N)}$ defined in (12) for all $i, N$ with $i \leq N$. Then the following hold:

1. $F_{i}^{(N)}(0)=0, \quad \forall N \in \mathbb{N}$ and $i=1, \ldots, N$.;

2. There exists $c>0$ such that $\left.|| F_{i}^{(N)}\right|_{\infty} \leq c$ for all $i, N, i \leq N$.

Proof. 1. For $i \geq 2$ we have

$F_{i}^{(N)}(0)=\lim _{s \rightarrow 0} \frac{S P(1-T) \Gamma^{i-2}}{1-\Gamma^{N}}=\Gamma(0)^{i-2} \lim _{s \rightarrow 0} S P \lim _{s \rightarrow 0} \frac{1-T}{1-\Gamma^{N}}$

Given that $Q(0)=1, T(0)=1$, we have that $\Gamma(0)=1$. Also, we have $S(0)=1-T(0)=0$ and $S^{\prime}(0)=T^{\prime}(0)$, and therefore $S$ has two zeros at $s=0$. Since $P$ has a single pole at $s=0$ we have that $\lim _{s \rightarrow 0} S P=0$. Now, $(1-T) /\left(1-\Gamma^{N}\right)$ is of the form $0 / 0$ when $s \rightarrow 0$ and the following limit can be computed using L'Hôpital's rule

$\lim _{s \rightarrow 0} \frac{1-T}{1-\Gamma^{N}}=\lim _{s \rightarrow 0} \frac{T^{\prime}}{N \Gamma^{N-1} \Gamma^{\prime}}$

Since $T^{\prime}(0)=0$ and $Q^{\prime}=h$ it follows that $\Gamma^{\prime}=T^{\prime} / Q-T Q^{\prime} / Q^{2}$ and $\Gamma^{\prime}(0)=-h$. With this, evaluating the last limit yields $F_{i}^{(N)}(0)=0$ for $i \geq 2$. For $F_{1}^{(N)}$ defined in (13) we have

$F_{1}^{(N)}(0)=\lim _{s \rightarrow 0} \frac{S P\left(\Gamma^{N-1}-Q\right)}{1-\Gamma^{N}}=\lim _{s \rightarrow 0} S P \lim _{s \rightarrow 0} \frac{\Gamma^{N-1}-Q}{1-\Gamma^{N}}$

The second limit is again of the form $0 / 0$ and using L'Hôpital's rule

$\lim _{s \rightarrow 0} \frac{\Gamma^{N-1}-Q}{1-\Gamma^{N}}=\lim _{s \rightarrow 0} \frac{(N-1) \Gamma^{N-2} \Gamma^{\prime}-h}{-N \Gamma^{N-1} \Gamma^{\prime}}=\frac{-N h}{N h}=-1$.

Since $\lim _{S \rightarrow 0} S P=0$, we have that $F_{1}^{(N)}(0)=0$.

2. First we consider $i \geq 2$ fixed. From (1) we have that $F_{i}^{(N)}(0)=0$. From Proposition 1 , we have ||$\Gamma \|_{\infty} \leq 1$, and for $\omega>0$

$\left|\frac{S P(1-T) \Gamma^{i-2}}{1-\Gamma^{N}}\right| \leq|S P|\left|\frac{1-T}{1-\Gamma^{N}}\right|$.

The product $S P$ is stable and proper by design, therefore ||$S P||_{\infty}$ $\leq c_{1}$ with $c_{1}>0$. Since $\lim _{s \rightarrow 0} \frac{1-T}{1-\Gamma^{N}}=0$, as seen in the previous point, and $|\Gamma|<1$ for all $\omega>0$, we have that $\left|\frac{1-T}{1-\Gamma^{N}}\right|$ is well defined for every $\omega \geq 0$. Now, since $|\Gamma|<1$ for $\omega>0$

$\left|\frac{1-T}{1-\Gamma^{N}}\right| \leq \frac{|1-T|}{1-|\Gamma|^{N}} \leq \frac{|1-T|}{1-|\Gamma|^{2}}$,

for all $\omega \geq 0$. Since $T(0)=1$ and $T^{\prime}(0)=0$, the factor $|1-T|$ has at least two zeros at $\omega=0$. We will show that $1-|\Gamma|^{2}$ does not have 
more than two zeros at $\omega=0$, given that $h>h_{0}$. In particular, since $\Gamma(0)=1,1-|\Gamma|^{2}$ has at least one zero at $\omega=0$. We will show that

$\left.\frac{d^{2}}{d \omega^{2}}(1-|\Gamma|)^{2}\right|_{\omega=0} \neq 0$.

Let $g_{1}(\omega)=|1 / Q|^{2} \quad$ and $\quad g_{2}(\omega)=|T|^{2} \quad$ which yields $1-|\Gamma|^{2}=1-g_{1}(\omega) g_{2}(\omega)$. We have that $g_{1}(\omega)=1 /\left(1+\omega^{2} h^{2}\right)$ and consequently $g_{1}^{\prime}(0)=0$. Assuming that $g_{2}^{\prime}(0)=0$ (otherwise would imply that $1-|\Gamma|^{2}$ has only one zero at $\omega=0$, and therefore there is nothing to prove), and given that $g_{1}(0)=g_{2}(0)=1$ we have that

$\left.\frac{d^{2}}{d \omega^{2}}(1-|\Gamma|)^{2}\right|_{\omega=0}=-g_{1}^{\prime \prime}(0)-g_{2}^{\prime \prime}(0)$.

In particular $g_{1}^{\prime \prime}(0)=-2 h^{2}$ and Lemma 2 states that

$2 h_{0}^{2} \geq \frac{d^{2}}{d \omega^{2}}|T|_{\omega=0}^{2}=g_{2}^{\prime \prime}(0)$.

By hypothesis $h>h_{0}$, and we have that $2 h^{2}>g_{2}{ }^{\prime \prime}(0)$. This implies that $1-|\Gamma|^{2}$ has at most two zeros at $\omega=0$. Consequently there exists $c_{2}>0$ independent of $n$ such that

$\frac{|1-T|}{\left|1-\Gamma^{N}\right|} \leq \frac{|1-T|}{1-|\Gamma|^{2}}<c_{2}$ for all $\omega \geq 0$.

It follows that $\left.|| F_{i}^{(N)}\right|_{\infty} \leq c_{1} c_{2}$ for all $n \in \mathbb{N}$ and $i \geq 2$.

Following a similar approach for $F_{1}^{(N)}$ defined in (13) we have

$\left|F_{1}^{(N)}\right|=\left|\frac{S P\left(\Gamma^{n-1}-Q\right)}{1-\Gamma^{N}}\right|=\left|\frac{S P\left(\Gamma^{n-1}-1+1-Q\right)}{1-\Gamma^{N}}\right|$.

By the triangle inequality

$\left|F_{1}^{(N)}\right| \leq\left|\frac{S P\left(\Gamma^{N-1}-1\right)}{1-\Gamma^{N}}\right|+\left|\frac{S P(1-Q)}{1-\Gamma^{N}}\right|$.

The second term in the right hand side of (30) satisfies for all $\omega \geq 0$

$\left|\frac{S P(1-Q)}{1-\Gamma^{N}}\right| \leq \frac{|\operatorname{sh} S P|}{1-|\Gamma|^{2}}$

where we used the fact $1-Q=-s h$. The factor $s h S P$ is proper and has two zeros at $s=0$ (since $S P$ is strictly proper and already has one zero at $s=0$ ). From the discussion for $i \geq 2$ we have that $1-$ । $\left.\Gamma\right|^{2}$ has at most two zeros at $\omega=0$, therefore we have that there exists $c_{31}>0$ independent of $N$ such that

$\left|\frac{S P(1-Q)}{1-\Gamma^{N}}\right| \leq c_{31} \quad$ for all $\omega \geq 0$.

For the first term in (30) we have

$$
\begin{aligned}
\left|\frac{S P\left(\Gamma^{N-1}-1\right)}{1-\Gamma^{N}}\right| & \leq \frac{|S P(\Gamma-1)|}{1-|\Gamma|} \frac{\left|1+\Gamma+\cdots+\Gamma^{N-2}\right|}{1+|\Gamma|+\cdots+|\Gamma|^{N-1}} \\
& \leq \frac{|S P(\Gamma-1)(1+|\Gamma|)|}{1-|\Gamma|^{2}} \frac{1+|\Gamma|+\cdots+|\Gamma|^{N-2}}{1+|\Gamma|+\cdots+|\Gamma|^{N-1}} .
\end{aligned}
$$

and using a similar reasoning to previous cases we have that $\mid S P$ $(\Gamma-1) \mid$ has at least two zeros at $\omega=0$ and $1-|\Gamma|^{2}$ has at most two zeros at $\omega=0$. Hence, there exists $c_{32}>0$ independent of $n$ such that

$\frac{|S P(\Gamma-1)(1+|\Gamma|)|}{1-|\Gamma|^{2}}<c_{32}$ for all $\omega \geq 0$.

Finally, using the fact

$\frac{1+|\Gamma|+\cdots+|\Gamma|^{n-2}}{1+|\Gamma|+\cdots+|\Gamma|^{n-1}} \leq 1$ for all $\omega \geq 0$,

we can conclude that there exists $c=c_{31}+c_{32}>0$ independent of $n, i$ such that

$\left|F_{1}^{(N)}\right| \leq c_{3}$ for all $\omega \geq 0$.

Part (1) of the previous theorem shows that the transfer functions $\left\{F_{i}^{(N)}\right\}$ have 0 DC-gain independent of the size of the string and the dynamics of the vehicles and controllers (besides the poles at the origin). Part (2) shows that the sequences $\left\{F_{i}^{(N)}\right\}$ are string stable. In other words, the effect of a disturbance on the first vehicle $D_{1}$ on the inter-vehicle spacing of the $i$ th vehicle $E_{i}=$ $X_{i-1}-X_{i}$ does not grow with an increase of the string size. Finally, Part (3) can be interpreted as the effect that a single disturbance to the first vehicle has on the inter-vehicle spacings of every vehicle is bounded independent of the string size.

\section{Cyclic interconnection with a leader}

In this section we consider the addition of an extra (possibly fictitious) vehicle, with position $z_{0}(t)$, that moves independently and such that every other member of the formation also tracks its position. We also consider a constant inter-vehicle spacing policy, that is $h=0$. If we let $\varepsilon_{i}^{0}=i \varepsilon$ be a fixed desired constant spacing from the $i$ th follower to the leader, then we define

$e_{i}^{0}(t)=z_{0}(t)-z_{i}(t)-i \varepsilon, \quad$ for $i=1, \ldots, N$,

$e_{i}(t)=z_{i-1}(t)-z_{i}(t)-\varepsilon, \quad$ for $i=2, \ldots, N$,

$e_{1}(t)=z_{N}(t)-z_{1}(t)+(N-1) \varepsilon$.

We consider initial positions such that the platoon starts at the desired formation, that is

$z_{i}(0)=-i \varepsilon, \quad$ for $i=0, \ldots, N$,

and we also consider the platoon to be initially at rest, i.e. $\dot{z}_{i}(0)=0$ for all $i=0, \ldots, N$.. Now, we use the change of coordinates

$x_{i}(t)=z_{i}(t)-z_{i}(0), \quad$ for $i=1, \ldots, N$,

which yields

$X_{i}=P\left(U_{i}+D_{i}\right), \quad$ for $i=0, \ldots, N$,

$E_{i}=X_{i-1}-X_{i}, \quad$ for $i=2, \ldots, N$,

$E_{1}=X_{N}-X_{1}$,

$E_{i}^{0}=X_{0}-X_{i}, \quad$ for $i=1, \ldots, N$.

The control strategy is now given by

$U_{i}=K\left(\eta E_{i}+(1-\eta) E_{i}^{0}\right), \quad$ for $2 \leq i \leq N$,

$U_{1}=K\left(\eta X_{N}+(1-\eta) E_{1}^{0}-\eta X_{1}\right)$

$U_{0}=0$,

where $\eta \in(0,1)$.

Remark 4. Note that the selection $U_{0}=0$ implies that the leader vehicle moves independently and its position is completely determined by its initial condition and the input signal $D_{0}$ as $X_{0}=P D_{0}$. Moreover, the control strategy is such that every follower aims to maintain a constant inter-vehicle spacings with respect to its immediate predecessor and with the leader.

Now we proceed in a similar fashion as in Section 3. With this control strategy, the vehicle dynamics are given by

$\underline{X}=\left(\mathbf{I}-P K \mathbf{G}_{0}\right)^{-1} P \underline{D}$, 
with $\underline{X}=\left[\begin{array}{llll}X_{0} & X_{1} & \cdots & X_{N}\end{array}\right]^{\top} \in \mathbb{C}^{N+1}$ and $\boldsymbol{G}_{0} \in \mathbb{C}^{N+1 \times N+1}$ being the new interconnection matrix:

$\boldsymbol{G}_{0}=\left[\begin{array}{cc}0 & \underline{0}^{\top} \\ (1-\eta) \underline{\boldsymbol{\Theta}} & \boldsymbol{\Theta}\end{array}\right]$

where $\underline{0} \in \mathbb{R}^{N}$ is the all zeros vector, $\underline{1} \in \mathbb{R}^{N}$ is the all ones vector, and

$\boldsymbol{\Theta}=\left[\begin{array}{cccc}-1 & & & \eta \\ \eta & \ddots & & \\ & \ddots & \ddots & \\ & & \eta & -1\end{array}\right]$.

Now we compute

$\left(\boldsymbol{I}-P K \boldsymbol{G}_{0}\right)^{-1}=\left[\begin{array}{cc}1 & \underline{0}^{\top} \\ -(1-\eta) P K \underline{1} & \boldsymbol{\Theta}_{0}\end{array}\right]^{-1}=\left[\begin{array}{cc}1 & \underline{0}^{\top} \\ (1-\eta) P K \boldsymbol{\Theta}_{0}^{-1} \underline{1} & \boldsymbol{\Theta}_{0}^{-1}\end{array}\right]$

with

$$
\begin{aligned}
\boldsymbol{\Theta}_{0}^{-1} & =\left[\begin{array}{cccc}
S^{-1} & & & -\eta P K \\
-\eta P K & \ddots & & \\
& \ddots & \ddots & \\
& & -\eta P K & S^{-1}
\end{array}\right]^{-1} \\
& =\frac{S}{1-(\eta T)^{N}}\left[\begin{array}{ccccc}
1 & (\eta T)^{N-1} & \cdots & (\eta T)^{2} & \eta T \\
\eta T & 1 & \ddots & & (\eta T)^{2} \\
\vdots & \ddots & \ddots & \ddots & \vdots \\
(\eta T)^{N-2} & & \ddots & 1 & (\eta T)^{N-1} \\
(\eta T)^{N-1} & (\eta T)^{N-2} & \cdots & \eta T & 1
\end{array}\right],
\end{aligned}
$$

for $S=1 /(1+P K)$ and $T=1-S$.

Remark 5. Note that in the following $\tilde{K}=K$ since $h=0$ and hence $T=P K /(1+P K)$.

Now, we define $\mathcal{F}_{i, k}^{(N)}$ as the transfer function from a disturbance $D_{k}$ at the $k$ th vehicle to the $i$ th inter-vehicle spacing $E_{i}$, which can be written as $E_{i}=X_{i-1}-X_{i}=\mathcal{F}_{i, k}^{(N)} D_{k}$. We have the following result.

Proposition 2. The transfer functions $\mathcal{F}_{i, k}$ satisfy

$\mathcal{F}_{1,0}^{(N)}=\frac{S P}{1-\eta T}$

$\mathcal{F}_{i, 0}^{(N)}=0$, for $i>1$.

Proof. Since the sum of every row of $\boldsymbol{\Theta}_{0}^{-1}$ in (53) is equal to $\frac{S}{1-(\eta T)^{N}} \sum_{i=0}^{N-1}(\eta T)^{i}$, we have that

$\boldsymbol{\Theta}_{0}^{-1} \underline{1}=\frac{S}{1-(\eta T)^{N}} \sum_{i=0}^{N-1}(\eta T)^{i} \underline{1}=\frac{S}{1-\eta T} \underline{1}$.

This implies that for $\underline{D}=\left[\begin{array}{llll}D_{0} & 0 & \cdots & 0\end{array}\right]^{\top}$,

$E_{1}=X_{0}-X_{1}=\left(1-(1-\eta) P K \frac{S}{1-\eta T}\right) P D_{0}=\frac{S P}{1-\eta T} D_{0}$,

and $E_{i}=X_{i-1}-X_{i}=0, \quad$ for $i>1$.

Remark 6. If we recall that $X_{0}=P D_{0}$ is the trajectory of the leader, we see that the every follower has the same transient when there are no other disturbances in the platoon. This can also be seen as all the followers moving as a unit when the only disturbance in the system is one at the leader. This is an interesting feature when using this interconnection.

The effect of a disturbance on the first follower, $D_{1}$, is given by $E_{1}=\mathcal{F}_{1,1}^{(N)} D_{1}=\frac{-S P}{1-(\eta T)^{N}} D_{1}$,
$E_{i}=\mathcal{F}_{i, 1}^{(N)} D_{1}=\frac{S P(1-\eta T)(\eta T)^{i-1}}{1-(\eta T)^{N}} D_{1}, \quad$ for $i>1$.

By the symmetry of the interconnection, the effect of $D_{k}$ with $k>1$ is the effect for $D_{1}$ shifted accordingly. Therefore, in the following we only study the transfer functions $F_{i, 1}^{(N)}$.

\subsection{Stability analysis}

In a similar way as in the leaderless cyclic case, the stability of the interconnection is determined by the stability of the transfer function $\frac{1-T}{1-(\eta T)^{N}}$. We have the following result.

Theorem 3. Let $T=P K /(1+P K)$ and $\eta \in(0,1)$. The following holds:

1. if $|\eta|<|| T||_{\infty}^{-1}$ then $(1-T) /\left(1-(\eta T)^{N}\right)$ has all of its poles in the open left half plane;

2. if $|\eta|>|| T||_{\infty}^{-1}$ then there exists $N_{c} \in \mathbb{N}$ such that for all $N>N_{c}$, $(1-T) /\left(1-(\eta T)^{N}\right)$ has poles in the right half plane.

Proof. 1. The condition $|\eta|<|| T||_{\infty}^{-1}$ implies that ||$\eta T||_{\infty}<1$ and moreover $\|(\eta T)^{N}||_{\infty}<1$. Hence, we have that $\left|1-(1-\eta T)^{N}\right|$ $<1$ for all $\omega \in \mathbb{R}$ and Rouche's Theorem ensures that $\left(1-(\eta T)^{N}\right)^{-1}$ is stable. Since $1-T$ is stable, we have that $(1-T) /\left(1-(\eta T)^{N}\right)$ has all of its poles in the open left half plane.

2. The condition $|\eta|>|| T||_{\infty}^{-1}$ implies that ||$\eta T||_{\infty}>1$ and the proof of Part (2) of Theorem 1 applies directly substituting $\Gamma$ for $\eta T$.

This result coincides with the sufficient conditions on $\eta$ for string stability in a unidirectional leader following scheme (See for example [19]). The case $\eta=\|T\|_{\infty}^{-1}$ will yield instability for the particular values of $N \in \mathbb{N}$ which satisfy the equation $1-\left(\eta T\left(j \omega_{c}\right)^{N}\right)$ $=0$ with $\omega_{c}$ being the frequency where $\left|T\left(j \omega_{c}\right)\right|=|| T||_{\infty}$.

\subsection{String stability analysis}

Following similar steps as in the analysis for string stability in the leaderless cyclic case, we obtain the following result for disturbances at the first follower $\left(D_{1} \neq 0\right)$ :

Theorem 4. Let $T=P K /(1+P K)$ and $\eta \in(0,1)$. Consider $\mathcal{F}_{i, 1}^{(N)}$ defined in (58) for all $i, N$ with $i \leq N$. Then the following hold:

1. $\mathcal{F}_{i, 1}^{(N)}(0)=0, \quad \forall i=1, \ldots, N$;

2. if $|\eta|<|| T||_{\infty}^{-1}$, then there exists $c>0$ such that || $\mathcal{F}_{i, 1}^{(N)}||_{\infty}<c$ for all $i, N \in \mathbb{N}, i \leq N$.

Proof. 1. Since $\eta<1$ and $T(0)=1$, and recalling from Part (1) of Theorem 1 that $S P$ has one zero at $s=0$, direct substitution of $s=0$ into (58) implies that $\mathcal{F}_{i, 1}^{(N)}(0)=0, \quad \forall i=1, \ldots, N$.

2. From (1) we have that $\mathcal{F}_{i, 1}^{(N)}(0)=0$. The condition $|\eta|<|| T||$ ${ }_{\infty}^{-1}$ implies that ||$(\eta T)^{k}||_{\infty}<1$ for all $k \geq 1$. Moreover, ||$S P||_{\infty}<$ $c_{1}$ (see Part (2) of Theorem 2) and $\left.||(1-\eta T)\right|_{\infty}=c_{2}$. Therefore

$\left|\frac{S P(1-\eta T)(\eta T)^{i-1}}{1-(\eta T)^{N}}\right| \leq \frac{c_{1} c_{2}}{\left|1-(\eta T)^{N}\right|}$.

Given that ||$\eta T||_{\infty}<1$, we have that $1-(\eta T(j \omega))^{N}=0$ has no solutions for $\omega \in \mathbb{R}$ and therefore

$\frac{c_{1} c_{2}}{\left|1-(\eta T)^{N}\right|}<\frac{c_{1} c_{2}}{\min _{\omega}\left|1-(\eta T(j \omega))^{N}\right|}<\frac{c_{1} c_{2}}{1-\left|\left(\eta T\left(j \omega_{c}\right)\right)\right|}$,

where $\omega_{c}$ satisfies $\left|T\left(j \omega_{c}\right)\right|=|| T||_{\infty}$. It follows that there exists $c>0$ such that

$\left.|| \mathcal{F}_{i, 1}^{(N)}\right|_{\infty}<c$ 
for all $i, N$ with

$c=\frac{c_{1} c_{2}}{1-\left|\left(\eta T\left(j \omega_{c}\right)\right)\right|}$.

Part (1) of the last result is analogous to Theorem 2 for the leaderless cyclic case. Part (2) shows that the interconnection is string stable for disturbances in any follower and also gives a bound for the maximum disturbance amplification. The case $\| \eta T$ $\|_{\infty}=1$ is not considered since it may yield instability for some values of $n$. In particular, if $\eta T=e^{j \phi}$ for some $\omega_{c}$, the factor

$\frac{1-\eta T}{1-(\eta T)^{N}}$,

in (58) may have an unstable pole at $\omega=\omega_{c}$ if $e^{j n \phi}=1$ and $e^{j \phi} \neq 1$.

\section{Numerical examples}

In this section we present numerical examples and simulations that illustrate the results of the paper. We consider the vehicle dynamics and local controllers

$P=\frac{1}{s(0.1 s+1)}, \quad \tilde{K}=\frac{2 s+1}{s(0.05 s+1)}$.

In Fig. 1 we have the magnitude plots of $\Gamma=P K /(1+P K Q)$ for different values of $h$. It can be noted that for increasing values of the time headway constant $h$, the magnitude peak decreases from ||$\Gamma \|_{\infty}=1.2103$ for $h=0$ to $\|\Gamma\|_{\infty}=1$ after some value of $h>0$. In particular we can compute $h_{0}$ defined in Proposition 1 as

$h_{0}=\sqrt{\sup _{\omega}\left(\frac{|P K /(1+P K)|^{2}-1}{\omega^{2}}\right)}=\sqrt{2} \approx 1.4142$.

This value is in agreement with Fig. 1 from where it can be estimated $h_{0}<2$. Now, according to Proposition 1 for $h>\sqrt{2}$ we will have $|\Gamma|<1$ for $\omega>0$.

\subsection{Stability analysis}

Leaderless case: In Fig. 2 the pole locations of the transfer function $\frac{1-T}{1-T^{N}}$, with $h=0$, are plotted for $N=3$ and $N=9$. For $N=3$ all the poles are in the open left half plane, however, for $N=9$ there is a pair of complex poles with positive real part. Part (2) of Theorem 1 predicts that instability occurs for large enough string size when $h=0$. For $h=2$, Fig. 3 shows a zoom into the locations of some of the poles of the transfer function $\frac{1-T}{1-\Gamma^{\mathrm{N}}}$. It can be seen that the poles remain to the left of the stability boundary for $N=20,50,100$. It can also be noted that for $N=100$ there exist two

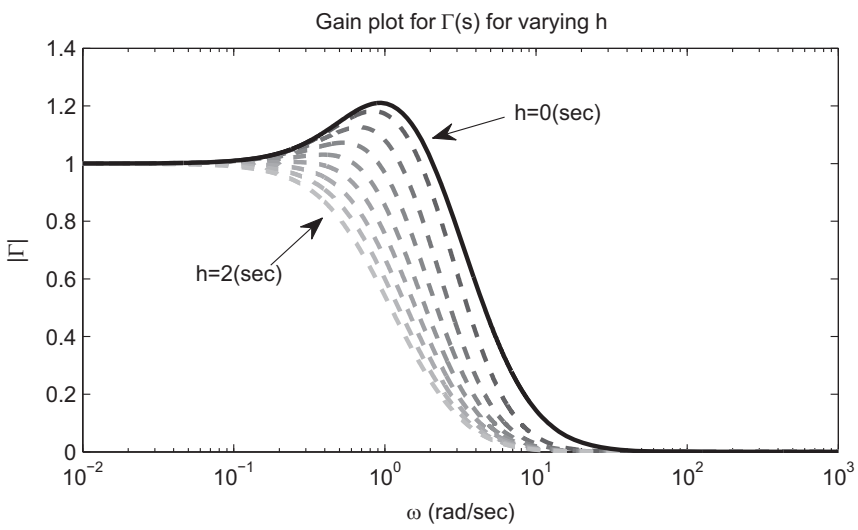

Fig. 1. Magnitude plots of $\Gamma=T /(1+s h)$ for different values of $h$. Solid line $h=0$ (s). Lightest gray and dashed line $h=2(s)$.

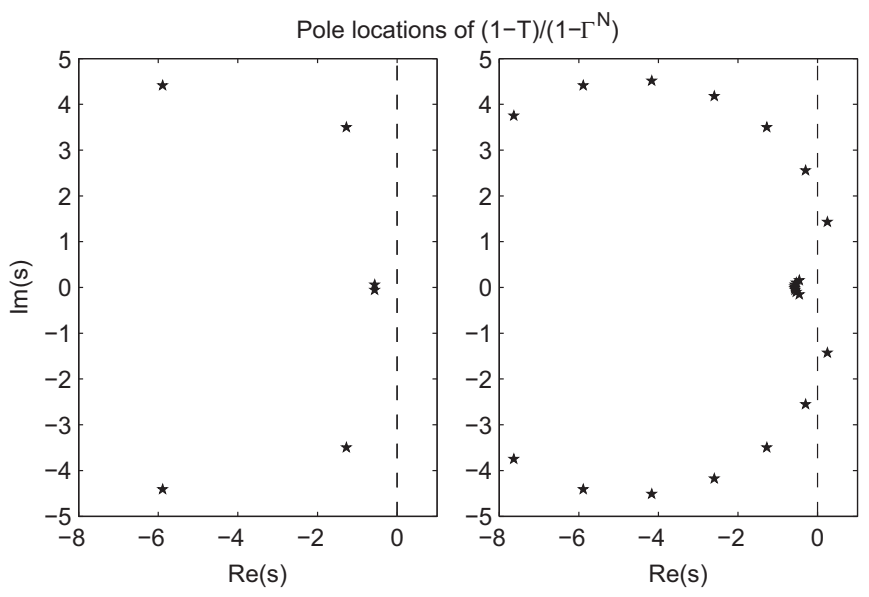

Fig. 2. Pole locations for $\frac{1-T}{1-\Gamma^{N}}$ with $h=0$ (s). Dashed line: stability boundary. Left: $N=3$. Right $N=9$.

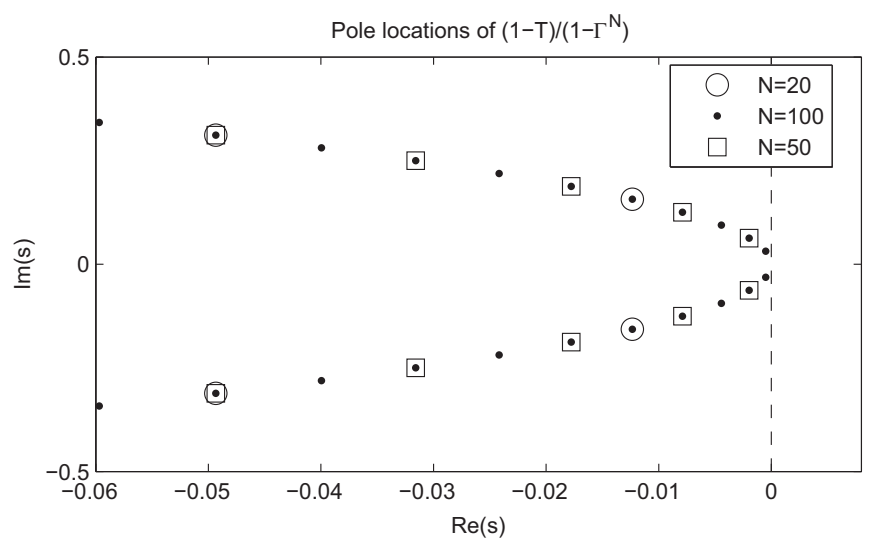

Fig. 3. Pole locations for $\frac{1-T}{1-\Gamma^{N}}$ with $h=2$ (s). Dashed line: stability boundary. Circles: $N=20$. Squares: $N=50$. Dots: $N=100$.
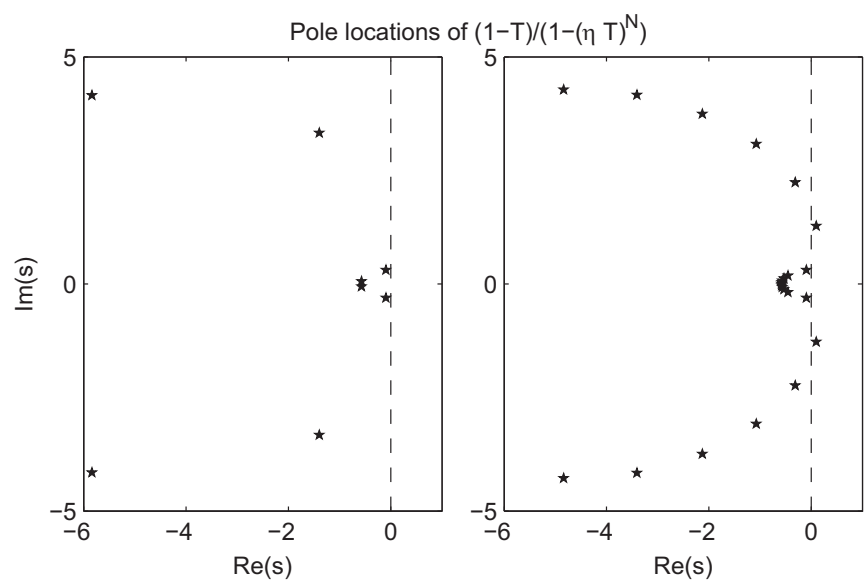

Fig. 4. Pole locations for $\frac{1-T}{1-(\eta T)^{N}}$ with $\eta=0.9$. Dashed line: stability boundary. Left: $N=3$. Right $N=9$.

poles that are closer to the boundary than the poles for $N=20,50$. It can be inferred that slower dynamics will occur with an increase of the string size. This can be predicted if we note that a factor $e^{j(2 k \pi / N)}-\Gamma$ tends to $1-\Gamma$ when $N$ grows large and $k=1$. Since $1-$ $\Gamma$ has a zero at $s=0$, slow poles for increasing $N$ should be expected.

Leader case: According to Theorem 3 the stability of the interconnection is ensured for $|\eta|<|| T||_{\infty}^{-1}$. In this particular case $\left.|| T\right|_{\infty} \approx 1.2$. Fig. 4 shows the pole locations of the transfer 


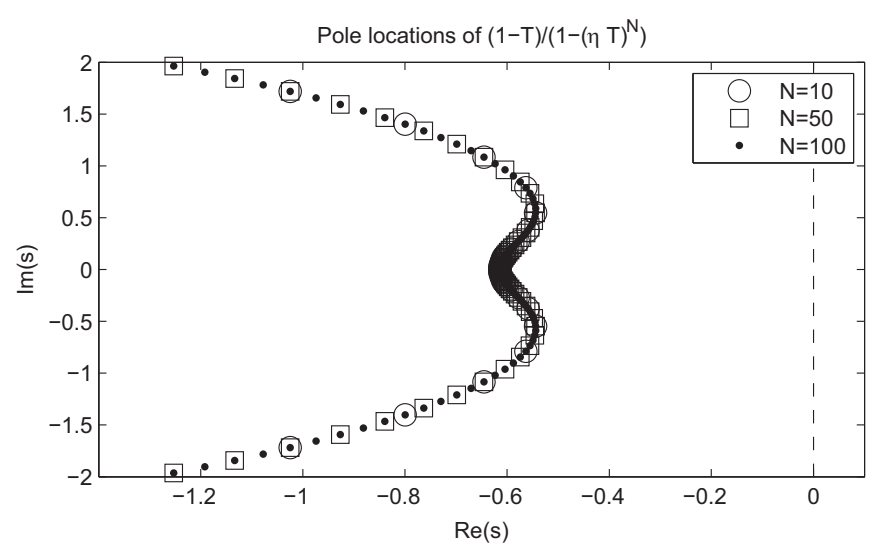

Fig. 5. Pole locations for $\frac{1-T}{1-\left(\eta T T^{N}\right.}$ with $\eta=0.5$. Dashed line: stability boundary. Circles: $N=10$. Squares: $N=50$. Dots: $N=100$.

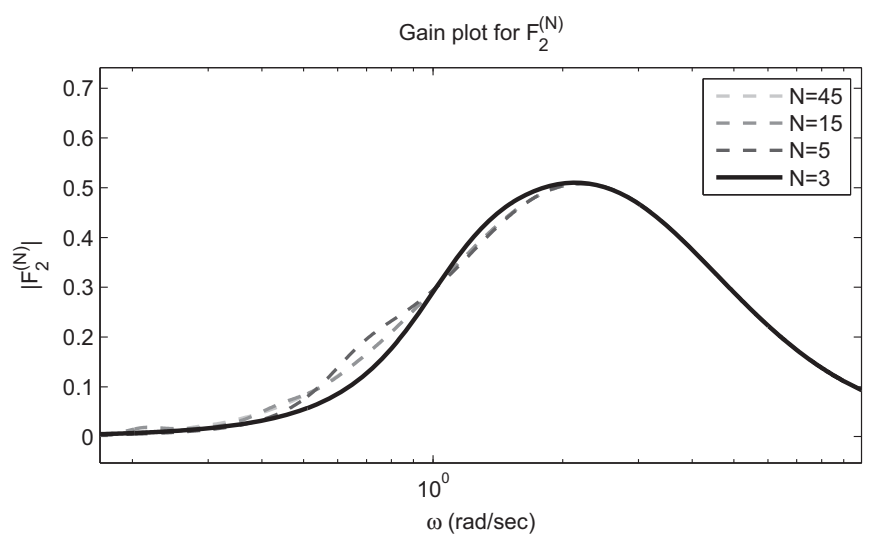

Fig. 6. Magnitude plots of $F_{2}^{(N)}$, when $h=2(\mathrm{sec})$, for an increasing number of vehicles.

function $\frac{1-T}{1-(\eta T)^{N}}$, with $\eta=0.9$, for $N=3$ and $N=9$. As predicted, since $\eta>1 / 1.2 \approx 0.83$, the system is unstable after the string size increases over a critical value. On the other hand, Fig. 5 shows the pole locations of $\frac{1-T}{1-(\eta T)^{N}}$ when $\eta=0.5$ for $N=20,50,100$. It can be seen that the system remains stable even for a string size as large as $N=100$. Moreover, the poles of the transfer function do not approach the stability boundary as the size of the string increases.

\subsection{String stability}

Leaderless case: The transfer functions $F_{2}^{(N)}$ reflect the effect of disturbances on the inter-vehicle spacing of the second member of the string with respect to its immediate predecessor, for different string sizes. For $h=2$, we obtain the corresponding magnitude plots, which are shown in Fig. 6. We can see that they are all bounded as predicted by Theorem 2 .

Leader case: The transfer functions $\mathcal{F}_{2,1}^{(N)}$ reflect the effect of disturbances on the inter-vehicle spacing of the second follower of the leader of the string with respect to its immediate predecessor, for different string sizes. For $\eta=0.5$, the corresponding magnitude plots are shown in Fig. 7. We can see that they are all bounded as predicted by Theorem 4 .

\section{Conclusions}

In this paper we provided stability and string stability results for a cyclic interconnection of vehicles. In particular we showed that if the spacing policy is constant, the formation becomes

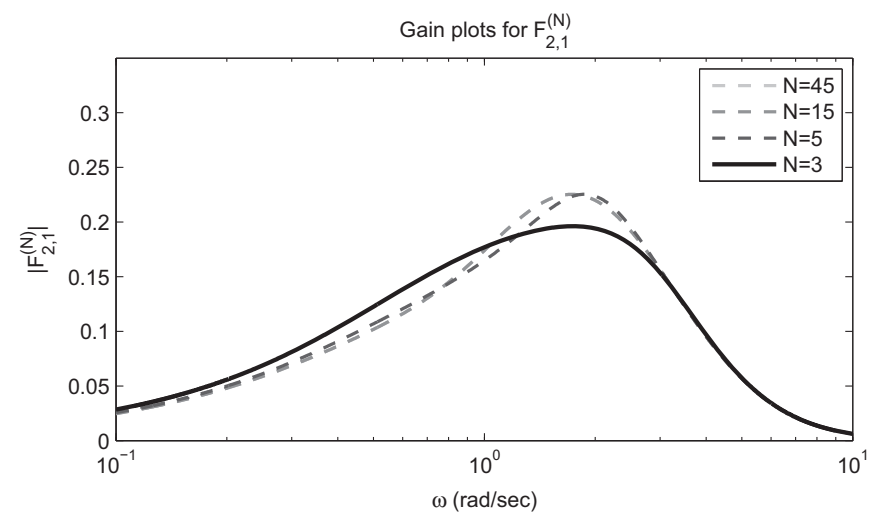

Fig. 7. Magnitude plots of $\mathcal{F}_{2,1}^{(N)}$, when $\eta=0.5$, for an increasing number of vehicles.

unstable for any string size greater than a critical value $N_{c}$. For the time headway spacing policy we show that it is possible to achieve stability and string stability of the interconnection, provided that the time headway constant is chosen appropriately.

Numerical examples illustrated these results and some of the drawbacks of the interconnection such as slower dynamics when the string size increases.

The use of an independent leader who is tracked by the remaining members of the vehicle string was also studied. We showed that similar results to the leaderless case apply, obtaining ensured stability for any string size and string stability when the design parameter $\eta\left\langle|| T||_{\infty}^{-1}\right.$. The added benefits are a simpler trajectory of the vehicles when the leader starts a manoeuvre and the dynamics do not become slower as the string size increases.

An immediate extension to this work is to study a cyclic and bidirectional interconnection with and without a leader. Other lines of work may include non-homogeneity of the vehicle models and controllers or the inclusion of time delays in the measurements. Moreover it would be of interest to study the effect of model uncertainty in all of this results.

\section{Acknowledgments}

This work is funded by the Irish Higher Education Authority (HEA), Telecommunications Graduate Initiative (TGI). The programme for research in Third level Institutions Cycle 5 is Cofunded by the Irish Government and the European Union under Ireland's EU Structural Funds Programme 2011-2015. This work has been (partially) funded by the Advanced Center for Electrical and Electronic Engineering, Basal Project FB0008, and by the Grant FONDECYT 3160738, CONICYT Chile.

\section{Appendix A. Proof of Lemma 3}

We write $\Gamma(s)=r(s) / m(s)$ where $r(s)$ and $m(s)$ are two polynomials without common factors and $m(s)$ is Hurwitz. Then, the solutions of $1-e^{j \theta} \Gamma=0$ are the roots of the polynomial $p(s)=m(s)-e^{j \theta} r(s)$. According to Proposition 3.4.5 in Hinrichsen and Pritchard [7], if a polynomial, $p(s)$, with complex coefficients is Hurwitz then

$\frac{d}{d \omega} \arg (p(j \omega))>0, \quad \forall \omega \in \mathbb{R}$.

We will show, given that $\Gamma$ is stable, strictly proper and ||$\Gamma \|_{\infty}>1$, that there exists an interval $\theta \in\left(\theta_{1}, \theta_{2}\right)$ with $\theta_{2}>\theta_{1}$ 
where condition (A.1) is violated. First, we write $p(j \omega)$ as

$p(j \omega)=|m(j \omega)| e^{j \psi_{m}(\omega)}\left(1+|\Gamma(j \omega)| e^{j \phi(\omega)}\right)$

where we factor $m(j \omega)=|m(j \omega)| e^{j \psi_{m}(\omega)}$ and add the arguments as $\phi(\omega)=\psi_{r}(\omega)-\psi_{m}(\omega)+\pi+\theta$. The argument of $p(j \omega)$ is

$$
\begin{aligned}
& \arg (p(j \omega))=\psi_{m}(\omega)+\arg \left(1+|\Gamma(j \omega)| e^{j \phi(\omega)}\right) . \\
& \quad=\psi_{m}(\omega)+\arg (1+|\Gamma(j \omega)|(\cos (\phi(\omega))+j \sin (\phi(\omega)))) .
\end{aligned}
$$

Computing the derivative using the formula of Proposition 3.4 .5 in Hinrichsen and Pritchard [7] (we omit the arguments $(\omega)$ for clarity)

$$
\frac{d}{d \omega} \arg (p(j \omega))=\mathfrak{R}\left\{\frac{p^{\prime}(j \omega)}{p(j \omega)}\right\}=\psi_{m}^{\prime}+\frac{|\Gamma|^{\prime} \sin (\phi)+|\Gamma| \phi^{\prime}(\cos (\phi)+|\Gamma|)}{(1+|\Gamma| \cos (\phi)) 2+|\Gamma|^{2} \sin ^{2}(\phi)} .
$$

Since $\Gamma(s)$ is strictly proper and ||$\Gamma \|_{\infty}>1$, there exists $\omega_{c}$ such that $|\Gamma|\left(\omega_{c}\right)=1$. If $|\Gamma|^{\prime}\left(\omega_{c}\right) \neq 0$ we compute the limit

$$
\begin{aligned}
\lim _{\omega \rightarrow \omega_{c}} \frac{d}{d \omega} \arg (p(j \omega)) & =\psi_{m}^{\prime}\left(\omega_{c}\right)+\frac{|\Gamma|^{\prime}\left(\omega_{c}\right) \sin \left(\phi_{c}\right)+\phi^{\prime}\left(\omega_{c}\right)\left(\cos \left(\phi_{c}\right)+1\right)}{2\left(1+\cos \left(\phi_{c}\right)\right)} \\
& =\psi_{m}^{\prime}\left(\omega_{c}\right)+\frac{\phi^{\prime}\left(\omega_{c}\right)}{2}+|\Gamma|^{\prime}\left(\omega_{c}\right) \frac{\sin \left(\phi_{c}\right)}{2\left(1+\cos \left(\phi_{c}\right)\right)}
\end{aligned}
$$

where $\phi_{c}=\lim _{\omega_{\rightarrow} \omega_{c}} \phi(\omega)$. In particular, the values $\psi_{m}^{\prime}\left(\omega_{c}\right), \phi^{\prime}\left(\omega_{c}\right)$ and $|\Gamma|^{\prime}\left(\omega_{c}\right)$ do not depend on the parameter $\theta$ and are bounded. However, the expression

$\frac{\sin \left(\phi_{c}\right)}{2\left(1+\cos \left(\phi_{c}\right)\right)}=\frac{\sin \left(\psi_{r}\left(\omega_{c}\right)-\psi_{m}\left(\omega_{c}\right)+\pi+\theta\right)}{2\left(1+\cos \left(\psi_{r}\left(\omega_{c}\right)-\psi_{m}\left(\omega_{c}\right)+\pi+\theta\right)\right)}$,

takes every possible value of the real numbers as $\theta$ varies. Therefore, there exists an interval $\left(\theta_{1}, \theta_{2}\right)$ with $\theta_{1}<\theta_{2}$ such that $\frac{d}{d \omega} \arg (p(j \omega))$ is negative, when $\omega=\omega_{c}$ and $\theta \in\left(\theta_{1}, \theta_{2}\right)$, which yields the desired result. If $|\Gamma|^{\prime}\left(\omega_{c}\right)=0$, we can only claim the existence of $\theta_{1}$ such that $1-e^{j \theta_{1}} \Gamma=0$ when $\omega=\omega_{c}$. Hence, the result follows with $\theta_{2}=\theta_{1}$.

\section{References}

[1] P. Barooah, J.P. Hespanha, Error amplification and disturbance propagation in vehicle strings with decentralized linear control, in: 44th IEEE Conference on
Decision and Control, 2005 and 2005 European Control Conference, CDCECC'05, IEEE, 2005, pp. 4964-4969.

[2] C.C. Chien, P. Ioannou, Automatic vehicle-following, in: American Control Conference, June 1992, 1992, pp. 1748-1752.

[3] K.C. Chu, Decentralized control of high-speed vehicular strings, Transport. Sci. 8 (4) (1974) 361-384.

[4] J. Fax, R. Murray, Information flow and cooperative control of vehicle formations, IEEE Trans. Automat. Control 49 (9) (2004) 1465-1476.

[5] H. Hao, P. Barooah, Stability and robustness of large platoons of vehicles with double integrator models and nearest neighbor interaction, Int. J. Robust Nonlinear Control 23 (18) (2013) 2097-2122.

[6] H. Hao, H. Yin, Z. Kan, On the robustness of large 1-d network of double integrator agents, in: American Control Conference (ACC), 2012, 2012, pp. 6059-6064.

[7] D. Hinrichsen, A. J. Pritchard, 2005. Mathematical systems theory I : modelling, state space analysis, stability and robustness, in: Texts in Applied Mathematics, Springer, New York, NY

[8] M.R. Jovanovic, B. Bamieh, On the ill-posedness of certain vehicular platoon control problems, IEEE Trans. Automat. Control 50 (9) (2005) 1307-1321.

[9] S. Klinge, R. Middleton, Time headway requirements for string stability of homogeneous linear unidirectionally connected systems, in: Proceedings of the 48th IEEE Conference on Decision and Control, 2009 held jointly with the 2009 28th Chinese Control Conference, CDC/CCC 2009, December 2009, pp. 1992-1997.

[10] G. Lafferriere, A. Williams, J. Caughman, J. Veerman, Decentralized control of vehicle formations, Syst. Control Lett. 54 (9) (2005) 899-910.

[11] W. Levine, M. Athans, On the optimal error regulation of a string of moving vehicles, IEEE Trans. Automat. Control 11 (July (3)) (1966) 355-361.

[12] F. Lin, M. Fardad, M.R. Jovanovic, Optimal control of vehicular formations with nearest neighbor interactions, IEEE Trans. Automat. Control 57 (9) (2012) 2203-2218.

[13] J. Marshall, M. Broucke, B. Francis, Formations of vehicles in cyclic pursuit, IEEE Trans. Automat. Control 49 (11) (2004) 1963-1974.

[14] R. Middleton, Trade-offs in linear control system design, Automatica 27 (2) (1991) 281-292.

[15] R. Middleton, J. Braslavsky, String instability in classes of linear time invariant formation control with limited communication range, IEEE Trans. Automat Control 55 (July (7)) (2010) 1519-1530.

[16] M.-C. Park, H.-S. Ahn, Stabilisation of directed cycle formations and application to two-wheeled mobile robots, IET Control Theory Appl. 9 (June (8)) (2015 1338-1346.

[17] D. Roberson, D. Stilwell, Decentralized control and estimation for a platoon of autonomous vehicles with a circulant communication network, in: American Control Conference, 2006, p. 6.

[18] J. Rogge, D. Aeyels, Vehicle platoons through ring coupling, IEEE Trans. Automat. Control 53 (6) (2008) 1370-1377.

[19] P. Seiler, A. Pant, K. Hedrick, Disturbance propagation in vehicle strings, IEEE Trans. Automat. Control 49 (10) (2004) 1835-1841.

[20] E. Shaw, J. Hedrick, String stability analysis for heterogeneous vehicle strings in: American Control Conference, 2007, ACC '07, July 2007, pp. 3118-3125.

[21] D. Swaroop, J. Hedrick, String stability of interconnected systems, IEEE Trans. Automat. Control 41 (March (3)) (1996) 349-357. 